\title{
Postdural Puncture Headache after Cervical Medial Branch Block
}

\section{Young In Lee, Hyo Jung Soh, Eung Don Kim}

Department of Anesthesiology and Pain Medicine, Daejeon St. Mary's Hospital, College of Medicine, The Catholic University of Korea, Daejeon, Korea

\begin{abstract}
Cervical medial branch block (MBB) is a frequently performed procedure for management of neck pain that rarely has complications. With fluoroscopic guidance, the procedure is considered a relatively safer procedure than epidural block. We report a case of a 27-year-old woman presenting with postural headache after cervical MBB. Dural penetration by inappropriate needle placement was suspected after reviewing fluoroscopic images of the procedure. After conservative treatment, including bed rest and analgesic treatment, the patient completely recovered without any neurological complications. Complications associated with MBB are rare and previous case reports have focused only on infection or vascular injection as etiologies. This is the first report of complications related to dural puncture after cervical MBB. Our findings suggest that misplacement of the block needle by inaccurate alignment of both sides of the cervical articular pillar, assessed by fluoroscopic view during the procedure, can result in dural injury.
\end{abstract}

Keywords: Medial branch block; Post-dural puncture headache; Complications

\section{INTRODUCTION}

Neck pain is common in the general population. Facet joints, paraspinal muscles and ligaments, and herniated nucleus pulposus are all possible sources of neck pain [1].

Medial branch block (MBB) is primarily performed for diagnostic purposes. However, repetitive infiltration of a local anesthetic agent has also been reported to provide therapeutic effects [2]. Indeed, cervical MBB is a frequently performed procedure for management of neck pain.

Although observational studies reported various rates of intravascular penetration during cervical $\mathrm{MBB}$ as $3.9 \%$ to $10.9 \%[3,4]$, complications associated with the procedure are otherwise generally rare [1]. Thus, MBB is considered relatively safe compared to other block procedures, such as facet joint injection or epidural block.

Unintentional spinal anesthesia and meningitis after facet joint blocks have been reported previously [5]. However, the possibility of dural penetration during cervical MBB is extremely rare because the target point of the procedure is the bony surface of the articular pillar. In fact, complication related to dural puncture during cervical MBB has never been reported as a complication. Here, we report a case of postdural puncture headache (PDPH) after cervical MBB.

\section{CASE REPORT}

A 27-year-old woman who complained of left side neck and trapezius area pain for a few days underwent left side cervical MBB at a private hospital. She did not have any sign or history of headache and did not have any psychiatric problem.

Cervical MBB was conducted under fluoroscopy guidance with the patient in the supine position. A few hours after the procedure, the patient complained of postural headache and nausea and revisited the private hospital and then she underwent cervical epidural injection of $2-3 \mathrm{~mL}$ of saline under fluoroscopic guidance to reduce her postural headache. That night, the patient visited the emergency room of Daejeon St. Mary's Hospital complaining of no symptom improvement and a new posterior neck pain and tingling sensation in both hands, and she was admitted to our pain center. The patient's headache and nausea worsened when sitting or standing and regressed when lying down. She was unable to eat 


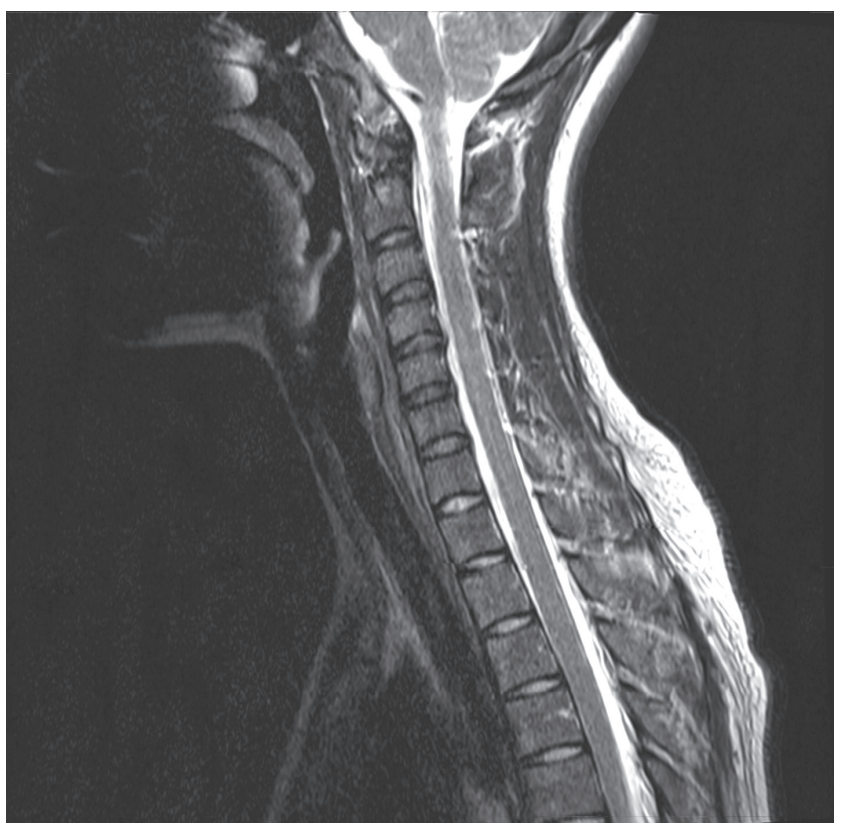

Fig. 1. Cervical magnetic resonance imaging of the patient. According to interpretation of a radiologic specialist, there was no evidence of dural injury except for mild disc bulging in $\mathrm{C} 3-4, \mathrm{C} 4-5$, and $\mathrm{C} 5-6$.

while sitting because of the postural headache. A cervical magnetic resonance imaging (MRI) was performed, which revealed no specific evidence of dural injury (Fig. 1).

The patient's tingling sensation spontaneously disappeared soon thereafter, and there were no specific neurologic abnormalities except for the postural headache. There were no symptoms suggesting infection, such as fever or chills, and laboratory findings were all within normal ranges.

According to the report of the physician of the private hospital who conducted the procedure, there was inappropriately deep placement of the needle. The needle did not contact any bony structure, and the physician did not inject any local agent after recognizing that the needle may have been misplaced. Furthermore, inaccurate alignments of articular pillars on both sides were found in fluoroscopic view of the procedure (Fig. 2). Thus, we assumed accidental dural penetration as a cause of the patient's postural headache despite of there was no definite finding of dural penetration on cervical MRI.

Intravenous fluid administration was initiated and bed rest was recommended. Acetaminophen and non-steroidal anti-inflammatory drugs were administrated to alleviate her headache. Two days later, the patient was able to eat while sitting and reported a $70 \%$ improvement of her headache. Four days after hospital ad-

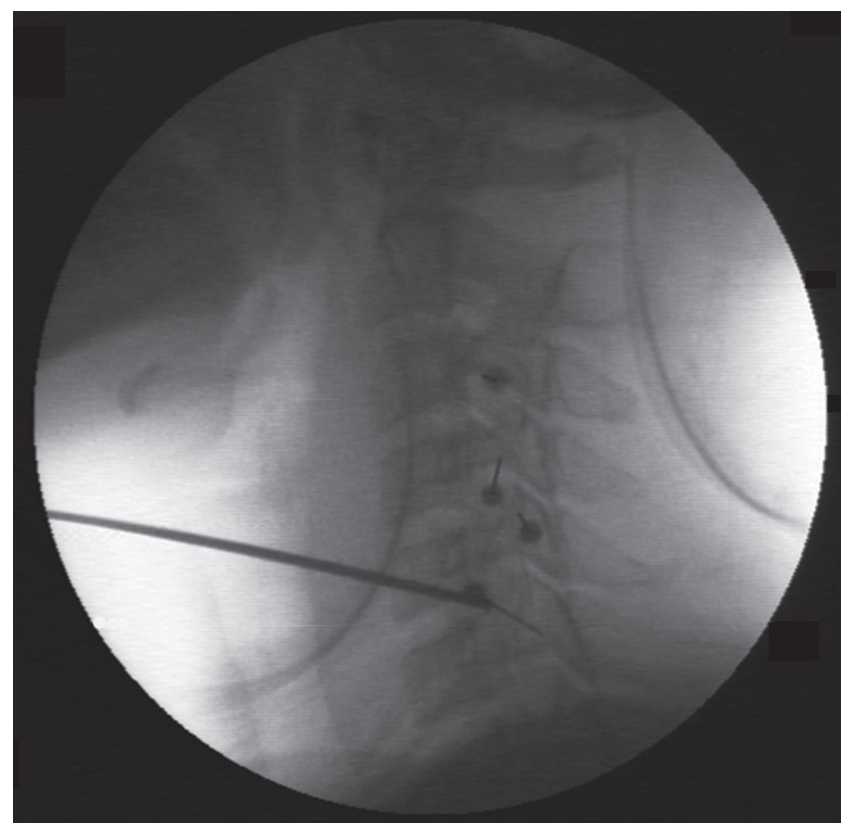

Fig. 2. Fluoroscopic lateral view during cervical medial branch block. The bony contours of articular pillars on both sides were not aligned at multiple levels. As a result of this inappropriate fluoroscopic view, the needle could pass through the facet joint instead of making contact with the articular pillar.

mittance, her headache had completely improved and the patient was discharged without any neurological sequelae. She reported that she was free of symptoms when followed up at 7 days after discharge.

\section{DISCUSSION}

Cervical facet joints below $\mathrm{C} 2-3$ are innervated by the medial branches from the cervical dorsal rami above and below level. The medial branches run around the waist of the articular pillar and are attached to periosteum by fascia [2].

Cervical MBB is generally performed under fluoroscopic guidance, and the target point of the block needle is the midpoint of the articular pillar of the cervical spine in the fluoroscopic lateral view [2]. After the needle makes contact with the bony structure, negative blood aspiration should be confirmed before local anesthetic agent injection.

The spinal cord is protected by bony structures, such as articular pillars, which are the target point of needle. Therefore, with regard to dural injury, $\mathrm{MBB}$ is generally considered a relatively safer procedure than epidural block.

However, inappropriate alignment of the bony contour of articular pillars on both sides in fluoroscopic view during the proce- 
dure can allow the needle to pass though the facet joint, resulting in dural penetration or nerve injury. Alignment of articular pillars on both sides in the fluoroscopic lateral view was not achieved in the present case.

Fortunately, a local anesthetic agent was not injected in the present case. If an anesthetic agent was injected without knowledge of the dural puncture during the cervical $\mathrm{MBB}$, there could have been catastrophic results.

The pathophysiology of PDPH involves decreases in intracranial pressure by cerebrospinal fluid leakage [6]. Epidural autologous blood injection is widely performed to reduce this pressure gradient. However, the viscosity of blood can cause space-occupying symptoms, such as radiating pain [7]. Therefore, some clinicians recommend epidural saline injection prior to epidural blood injection [8]. Less than $5 \mathrm{~mL}$ of volume is generally required in cases of cervical epidural blood patch; however, only few a milliliters of fluid can result in unnecessary neurological symptoms such as tingling sensation in both hands and newly developed posterior neck pain which were seen in the present case.

There is no consensus about timing for conducting an epidural blood or saline patch. However, there are several reports suggesting a greater than $50 \%$ recurrence rate of the headache if the epidural blood patch is conducted within the first 24 hours [6,9]. Therefore, earlier epidural blood patch, i.e., within the first 24-42 hours after dural puncture, may be less effective. In the present case, an early epidural saline injection within a few hours did not improve PDPH symptoms, which is in agreement with these aforementioned studies [6,9]. In present case, the patient's symptoms significantly improved over 2 days with conservative treatment. Therefore, there was no need for another epidural injection of saline or autologous blood.

Previous case reports of complications related to $\mathrm{MBB}$ or facet joint block have focused on infection or transient paraplegia due to intravascular injection $[10,11]$. In present case, postural headache was developed before the epidural saline injection and intensity of the headache was unchanged regardless of the epidural saline injection. Therefore, we believe that the cause of PHPD in present case was misplacement of needle during cervical MBB. To the best of our knowledge, the present case is the first report of complications of dural injury related to MBB.

In conclusion, our study suggests that when performing cervical MBB under fluoroscopic guidance, exact alignment of the articular pillar and minimizing the parallax of both sides is essential for a safe procedure. The present report may sound the alarm about dural injury during MBB with inadvertent fluoroscopic adjustment. In cases where obtaining an exact fluoroscopic view is difficult, alternative methods, such as ultrasound, can be considered to avoid dural injury.

\section{REFERENCES}

1. Van Eerd M, Patijn J, Lataster A, Rosenquist RW, van Kleef M, Mekhail N, et al. 5. Cervical facet pain. Pain Pract 2010;10:113-23.

2. Manchikanti L, Singh V, Falco FJ, Cash KM, Fellows B. Cervical medial branch blocks for chronic cervical facet joint pain: a randomized, doubleblind, controlled trial with one-year follow-up. Spine (Phila Pa 1976) 2008;33:1813-20.

3. Verrills P, Mitchell B, Vivian D, Nowesenitz G, Lovell B, Sinclair C. The incidence of intravascular penetration in medial branch blocks: cervical, thoracic, and lumbar spines. Spine (Phila Pa 1976) 2008;33:E174-7.

4. Jeon YH, Kim SY. Detection rate of intravascular injections during cervical medial branch blocks: a comparison of digital subtraction angiography and static images from conventional fluoroscopy. Korean J Pain 2015; 28:105-8.

5. Boswell MV, Colson JD, Sehgal N, Dunbar EE, Epter R. A systematic review of therapeutic facet joint interventions in chronic spinal pain. Pain Physician 2007;10:229-53.

6. Bezov D, Lipton RB, Ashina S. Post-dural puncture headache: part I diagnosis, epidemiology, etiology, and pathophysiology. Headache 2010;50: 1144-52.

7. Jo D, Kim ED, Oh HJ, Oh JY. Radicular pain followed by epidural blood patch. Pain Med 2014;15:1642-3.

8. Kakinohana M, Odo Y, Matsuda S, Taira Y, Sugahara K. Epidural injection with saline for treatment of postspinal headache: comparison with epidural blood patch. J Anesth 2001;15:185-7.

9. Paech MJ, Doherty DA, Christmas T, Wong CA; Epidural Blood Patch Trial Group. The volume of blood for epidural blood patch in obstetrics: a randomized, blinded clinical trial. Anesth Analg 2011;113:126-33.

10. Heckmann JG, Maihofner C, Lanz S, Rauch C, Neundorfer B. Transient tetraplegia after cervical facet joint injection for chronic neck pain administered without imaging guidance. Clin Neurol Neurosurg 2006;108:70911 .

11. Michel-Batot C, Dintinger H, Blum A, Olivier P, Laborde F, BettembourgBrault I, et al. A particular form of septic arthritis: septic arthritis of facet joint. Joint Bone Spine 2008;75:78-83. 